\title{
Alteraciones hematológicas en COVID-19
}

\author{
Hematological Findings in COVID-19
}

María Isabel Villa Palacioํㅜㄹ Elizabeth López Henao²

\section{Resumen}

El SARS (síndrome respiratorio agudo grave) es el estadio grave de la COVID-19 ocasionado por el SARS-CoV-2, que causa infecciones respiratorias en humanos y cuya transmisión se da principalmente por contacto. El virus ingresa a la célula huésped por la interacción de la proteína $S$ con la enzima convertidora de angiotensina II (ACE2), presente en el tracto respiratorio, así como en monocitos, macrófagos, células endoteliales, corazón y tracto gastrointestinal. El aumento de IFN frena la replicación viral y activa la respuesta inmune adaptativa. Así, las manifestaciones clínicas de la infección se presentan frecuentemente a nivel del tracto respiratorio; sin embargo, también pueden involucrar otros sistemas como el hematopoyético. En el hemograma se observan recuentos celulares alterados, principalmente leucocitos y plaquetas. La linfopenia y neutrofilia se asocian con enfermedad severa y la trombocitopenia se presenta de manera heterogénea en la infección. Entre las complicaciones se encuentra la coagulación intravascular diseminada, producida cuando los monocitos y las células endoteliales son activadas por la liberación de citoquinas; esto genera dańo endotelial, con la síntesis del factor tisular, secreción de factor tisular, activación plaquetaria y liberación del factor Von Willebrand, así como una condición hiperfibrinolítica especialmente en estadios tardíos de la infección. Las pruebas de laboratorio como el dímero $\mathrm{D}$, los productos de degradación de la fibrina (PDF), tiempo de protrombina (TP), tiempo de tromboplastina parcial activado (TTPA), entre otras, son fundamentales para el diagnóstico, seguimiento y pronóstico de la enfermedad.

Palabras claves: hematología, coagulación intravascular diseminada, hemostasia, fisiopatología, anticuerpos, linfopenia.

\footnotetext{
1. M. Sc. en Microbiología y Bioanálisis. Docente del área de Hematología. Institución Universitaria Colegio Mayor de Antioquia. ORCID: https://orcid.org/0000-0001-8645-692X

2. Bacterióloga y laboratorista clínica. M. Sc. en Educación Superior en Salud. ORCID: https://orcid.org/0000-0002-0143-6733

Correo electrónico de correspondencia: mariaisabelv@colmayor.edu.co
}

https://doi.org/10.22490/24629448.4189 


\section{Abstract}

Severe Acute Respiratory Syndrome (SARS) is the serious condition of coronavirus (COVID-19) caused by SARS-COV-2 which causes respiratory infections in humans, and whose transmission is given mainly through the contact. this virus enters into the host cell due to the spike protein $(S)$ interaction with the angiotensin-converting enzyme 2 (ACE2), which is not only present in the respiratory tract but also monocytes, macrophages, endothelial cells, the heart, and gastrointestinal tract. The increase in INF stops viral replication and activates the adaptive immune response. The infection's clinic manifestations often occur in the respiratory tract; however, other systems like the hematopoietic may be affected. Altered cell counts, mainly leukocytes and platelets, are seen on the blood count. Lymphopenia and neutrophilia are associated with severe disease; thrombocytopenia is present in a heterogeneous way in the infection. Among the disease's complications are the Disseminated Intravascular Coagulation (DIC) that results when monocytes and endothelial cells are activated because of the release of cytokines, causing endothelial damage, with the synthesis of the tissue factor, tissue factor discharge, platelet activation, and the von Willebrand factor release, generating a hyperfibrinolytic condition especially in the infection's late-stage. Laboratory tests such as D-dimer (D-D), Fibrinogen Degradation Products (FDP), Prothrombin Time (PT), Activated Partial Thromboplastin Time (APTT) among others, are essential to the diagnosis, monitoring, and prognosis of the disease.

Keywords: Hematology, disseminated intravascular coagulation, coronavirus, hemostasis, physiopatology,antibodies, Lymphopenia.

\section{Patogénesis y respuesta inmune en infección por SARS-CoV 2}

El SARS (síndrome respiratorio agudo grave) es el estadio grave de la COVID-19 ocasionado por el SARS-CoV-2, virus respiratorio que pertenece a la familia Coronaviridae, subfamilia Orthocoronaviridae. Es un virus RNA monocatenario de polaridad positiva, cuyo genoma es de 26 a $32 \mathrm{~Kb}$ y hace parte del grupo de los betacoronavirus que causan infección respiratoria en huma- nos, con transmisión principalmente por contacto $(1,2)$. El virus SARS-CoV 2 ingresa a la célula huésped por la interacción de la proteína $S$ con la enzima convertidora de angiotensina II ACE2, exopeptidasa de membrana presente en células alveolares tipo 2 del pulmón, así como en monocitos, macrófagos, células endoteliales, corazón y tracto gastrointestinal $(1,3,4,5,6)$. La activación de la vía de IFN frena la replicación viral y activa la respuesta inmune adaptativa; se genera un incremento exacerbado 
de citocinas proinflamatorias, denominada "tormenta de citoquinas", de manera que hay incremento en los niveles de interleucinas como IL-6, IL-2, IL-7, G-CSF, IFN-Y y TNF que parece estar implicado en la apoptosis linfocitaria $(3,4,7)$.

Por su parte, los linfocitos T CD4+ promueven la producción de anticuerpos específicos del virus mediante la activación de linfocitos $\mathrm{B}$ dependientes de $\mathrm{T}$; los CD8+ son citotóxicos y pueden eliminar directamente las células infectadas. La disminución de las células TCD4+ se asocia con una captación de linfocitos a nivel pulmonar, así como la producción de citoquinas y anticuerpos reducida. De esta manera, se genera un cuadro de neumonía grave conocido como síndrome respiratorio severo que consiste en inflamación, daño tisular, deterioro funcional del pulmón, falla multiorgánica, shock $y$, en casos severos, muerte $(4,8,9)$.

\section{Alteración de los valores del hemograma}

Las manifestaciones de la infección por SARS-CoV 2 se presentan principalmente a nivel del tracto respiratorio; sin embargo, pueden involucrar otros sistemas como el hematopoyético. Las personas con comorbilidades tienen un mayor riesgo de complicaciones, entre ellas, miocarditis fulminante y coagulación intravascular diseminada (10).

Una de las pruebas utilizadas para el apoyo diagnóstico de la enfermedad es el he- mograma, en el cual se observan recuentos celulares alterados, principalmente leucocitos y plaquetas. Los leucocitos pueden estar disminuidos con valores totales en casos severos menores de $<2 \times 10^{9} / \mathrm{L}$. La linfopenia se presenta de forma moderada o severa con valores absolutos de e 0,5-1 × 109/L y $<0,5$ $\times 109 / \mathrm{L}$, respectivamente, y se asocia con un riesgo mayor de desarrollar síndrome de distrés respiratorio agudo (ARDS) al igual que una probabilidad mayor de gravedad e ingreso a unidad de cuidados intensivos $(4,11)$.

En el extendido de sangre periférica es común observar la presencia de linfocitos reactivos con características plasmocitoides. Los neutrófilos en pacientes con enfermedad severa pueden presentar valores absolutos de 11,6 x 109/L (12). La morfología reportada en la línea granulocítica comprende hipergranulación, hiposegmetación e hipercondensación nuclear, así como la posibilidad de hipersegmentación $(13,14)$.

Finalmente, los datos relacionados con el recuento plaquetario son muy heterogéneos; algunos han sugerido su asociación con un curso desfavorable de la enfermedad y pueden estar entre 100000 y 150000 / $\mathrm{mm}^{3}(15)$.

\section{Covid 19 y trastornos de la coagulación}

La coagulación intravascular diseminada es una condición clínica secundaria de 
enfermedades de base, traumas, neoplasias y sepsis. La infección viral causada por el COVID-19 produce sepsis, al activar una fuerte respuesta inflamatoria sistémica que genera un desbalance en la homeostasis $(16,17,18)$.

La disfunción de la célula endotelial resulta de la liberación de interleucinas, principalmente la IL 6, potente proinflamatoria que induce la expresión del factor tisular en células endoteliales y monocitos, así como la síntesis del fibrinógeno, producción plaquetaria y liberación del factor Von Willebrand. La circulación libre de trombina y la alteración de los anticoagulantes naturales generan un estado de hipercoagulabilidad, en el cual se observa agregación en la microy macrovasculatura, y secundaria activación de una condición hiperfibrinolítica en estadios tardíos de la infección.

Adicionalmente, la hipoxia que está presente en los pacientes con neumonía puede estimular la trombosis, no solo a través del aumento de la viscosidad celular, sino por el aumento del factor inducible de hipoxia (HIF-1/HIF-2) $(16,19,20,21)$. La alteración del sistema de la hemostasia incluye cambios en el tiempo de tromboplastina parcial activado (PTTa) y en el tiempo de protrombina (TP), y los productos de degradación de la fibrina (PDF) y el dímero $\mathrm{D}$ se encuentran moderada o marcadamente aumentados, principalmente en pacientes con enfermedad severa (22). Finalmente, se plantea la posibilidad de encontrar anticuerpos antifosfolípidos en pacientes con la enfermedad (23). De hecho, recientemente se planteó la posible relación de los antígenos del sistema $A B O$ con la infección por COVID-19, lo que sugiere que el grupo sanguíneo A es más susceptible que los otros antígenos del sistema, de lo que se infiere el posible papel protector del grupo $\mathrm{O}(24)$.

\section{Referencias}

1. Lozada-Requena I, Ponce CN. COVID-19: respuesta inmune y perspectivas de intervenciones terapéuticas. Rev Peru Med Exp Salud Publica. 2020;37(2):312-9.

2. Wu H. E. Síndrome Respiratorio Agudo Severo. Revista Chilena de Pediatria. 2003.

3. Lin L, Lu L, Cao W, Li T. Hypothesis for potential pathogenesis of SARS-CoV-2 infection-a review of immune changes in patients with viral pneumonia. Emerg Microbes Infect. 2020;9(1):727-32.

4. Terpos E, Ntanasis-Stathopoulos I, Elalamy I, Kastritis E, Sergentanis TN, Politou M, et al. Hematological findings and complications of COVID-19. Am J Hematol. 2020;95(7):834-47.

5. García-Salido A. Narrative review of the immune response against coronavirus: An overview, applicability for SARS-COV-2, and therapeutic implications. An Pediatr [Internet]. 2020;93(1):60. e1-60.e7. DOI: https://doi.org/10.1016/j.anpedi.2020.04.016

6. El E, Nl H-, Severo RA, Oriente M, Oriente M, Cov S-. Revisión breve sobre COVID-19. Osmosis Rev Medica Estud. 2020;1(12):8. Available from: https://wdg.biblio.udg.mx/COVID19/ bayro2020editorial.pdf

7. Marietta M, Ageno W, Artoni A, De Candia E, Gresele P, Marchetti M, et al. COVID-19 and haemostasis: A position paper from Italian Society on Thrombosis and Haemostasis (SISET). Blood Transfus. 2020;18(3):167-9. 
8. Li G, Fan Y, Lai Y, Han T, Li Z, Zhou P, et al. Coronavirus infections and immune responses. J Med Virol. 2020;92(4):424-32.

9. Walls AC, Park YJ, Tortorici MA, Wall A, McGuire AT, Veesler D. Structure, Function, and Antigenicity of the SARS-CoV-2 Spike Glycoprotein. Cell [Internet]. 2020;181(2):281-292.e6. DOI: https:// doi.org/10.1016/j.cell.2020.02.058

10. Yang X, Yu Y, Xu J, Shu H, Xia J, Liu H, et al. Clinical course and outcomes of critically ill patients with SARS-CoV-2 pneumonia in Wuhan, China: a single-centered, retrospective, observational study. Lancet Respir Med [Internet]. 2020;8(5):475-81. DOI: https://doi.org/10.1016/ S2213-2600(20)30079-5

11. Tan L, Wang Q, Zhang D, Ding J, Huang Q, Tang $Y Q$, et al. Lymphopenia predicts disease severity of COVID-19: a descriptive and predictive study. Signal Transduct Target Ther. 2020;5(1):16-8.

12. Fan BE, Chong VCL, Chan SSW, Lim GH, Lim KGE, Tan GB, et al. Hematologic parameters in patients with COVID-19 infection. Am J Hematol. 2020;95(6):E131-4.

13. Zini G, Bellesi S, Ramundo F, d'Onofrio G. Morphological anomalies of circulating blood cells in COVID-19. Am J Hematol. 2020;95(7):870-2.

14. Salib C, Teruya-Feldstein J. Hypersegmented granulocytes and COVID-19 infection. Blood. 2020;135(24):2196.

15. Guan W, Ni Z, Hu Y, Liang W, Ou C, He J, et al. Clinical characteristics of coronavirus disease 2019 in China. N Engl J Med. 2020;382(18):1708-20.

16. Ranucci M, Ballotta A, Di Dedda U, Bayshnikova E, Dei Poli M, Resta M, et al. The procoagulant pattern of patients with COVID-19 acute respiratory distress syndrome. J Thromb Haemost. 2020;(March):1-5.

17. Weiss SR, Navas-Martin S. Coronavirus Pathogenesis and the Emerging Pathogen Severe Acute Res- piratory Syndrome Coronavirus. Microbiol Mol Biol Rev. 2005;69(4):635-64.

18. Ding R, Wang Z, Lin Y, Liu B, Zhang Z, Ma X. Comparison of a new criteria for sepsis-induced coagulopathy and International Society on Thrombosis and Haemostasis disseminated intravascular coagulation score in critically ill patients with sepsis 3.0: A retrospective study. Blood Coagul Fibrinolysis. 2018;29(6):551-8.

19. Connors JM, Levy JH. COVID-19 and its implications for thrombosis and anticoagulation. Blood. 2020;135(23):2033-40.

20. Tang N, Li D, Wang X, Sun Z. Abnormal coagulation parameters are associated with poor prognosis in patients with novel coronavirus pneumonia. J Thromb Haemost. 2020;18(4):844-7.

21. Yin S, Huang M, Li D, Tang N. Difference of coagulation features between severe pneumonia induced by SARS-CoV2 and non-SARS-CoV2. J Thromb Thrombolysis. 2020;3-6. DOI: https:// doi.org/10.1007/s11239-020-02105-8

22. Violi F, Pastori D, Cangemi R, Pignatelli P, Loffredo L. Hypercoagulation and Antithrombotic Treatment in Coronavirus 2019: A New Challenge. Thromb Haemost. 2020;120(6):949-56.

23. Yan Zhang, M.D. Meng Xiao, M.Sc. Shulan Zhang, M.D. Peng Xia MD, Wei Cao MD, Wei Jiang MD, Huan Chen MD, Xin Ding MD, Hua Zhao, M.D. Hongmin Zhang MD. Correspondence Coagulopathy and Antiphospholipid Antibodies in Patients with Covid-19. Nejm. 2020;38(1):1-3.

24. Ellinghaus D, Degenhardt F, Bujanda L, Buti $\mathrm{M}$, Albillos A, Invernizzi $\mathrm{P}$, et al. Genomewide Association Study of Severe Covid-19 with Respiratory Failure. N Engl J Med. 2020;NEJMoa2020283. http://www.nejm.org/doi/10.1056/ NEJMoa2020283 